\title{
CONFIANZA Y NORMA SOCIAL EN LA CORTESÍA LINGÜÍSTICA
}

\author{
J. A. G. Ardila \\ Universidad de Edimburgo \\ j.ardila@ed.ac.uk
}

\begin{abstract}
Resumen
Los estudios de cortesía atribuyen al español un modelo de cortesía positiva $y$, algunos, le imputan los efectos de la denominada confianza, esto es, del fenómeno etnolingüístico merced al cual los hablantes procuran adoptar unos hábitos lingüisticos propios de un contexto familiar, so pretexto de mostrar una actitud positiva. Este artículo pretende contender la tesis de que el español peninsular se rige por un modelo de cortesia que procura transmitir confianza. Luego de revisar la bibliografia al respecto, se cuestionan las hipótesis respecto de la confianza y se cotejan algunos usos pragmalingüísticos españoles con el inglés, el danés y el portugués: con el inglés y el danés puesto que, según estudios realizados, en contextos de formalidad, ingleses y daneses valoran la actitud positiva (léase colaborativa) de sus interlocutores sobre cualesquiera otras cualidades; con el portugués a fin de demostrar que los usos españoles no son comunes a otras lenguas romances. Vistas las discrepancias del español con estas otras lenguas, se aboga por trascender las conclusiones anteriores, para lo cual se propone la social norm view de Fraser.
\end{abstract}

PALABRAS CLAVE: imagen, confianza.

\begin{abstract}
Research suggests that Spanish has a model of positive politeness. Some linguists have further argued that Spanish politeness is amenable to the socalled confianza, or trustfulness - i.e. the ethnolinguistic phenomenon which compels the speakers to use familiar/colloquial rhetorical strategies, so as to display a positive attitude. This paper seeks to discuss the naturalisation of such confianza in Spanish linguistics; it will compare a number of pragmalinguistic examples in Spanish, English, Danish and Portuguese - according to previous cross-cultural research, English and Danish professional negotiators value positive attitude before any other criteria; a comparison with Portuguese will also prove that Spanish politeness does not at all coincide with politeness in other romance languages. In the light of the pragmalinguistic differences pinpointed, this paper will suggest that Spanish politeness studies should allow for Fraser's social norm view.
\end{abstract}

KEY WORDS: Face, confianza, Social Norm View.

\section{Introducción}

Las aportaciones al estudio de la cortesía lingüística en español suelen trazar con excesiva cautela las conclusiones que reflejan aquello que las aproximaciones empíricas parecen confirmar. Puesto que los análisis empíricos recogen la escasa recurrencia en español de fórmulas de cortesía (y especialmente de marcadores de cortesía), los lingüistas acostumbran a adoptar las siguientes dos posturas: primero, que es improcedente motejar una lengua (y, por ende, la cultura que la habla) de menos cortés que otra (v.g. Hickey y Vázquez Orta, 1994; Ballesteros, 2001), y, segundo, que la ausencia de fórmulas de cortesía en español se debe, en buena medida, al fenómeno sociopragmático que Bravo (1999) denominó confianza (vid. infra) (en lo sucesivo, se emplearán cursivas para distinguir la confianza como el término 
lingüístico forjado por Bravo de la confianza tal como se entiende en lengua española). La actual deriva de los estudios de cortesía descubre la necesidad de trascender estas dos posturas, cuya repetición, además de constituir ya un lugar común, muy bien pudiera reprimir el entendimiento de otras cuestiones. A tal fin, este trabajo revisa la confianza y demuestra que su aplicación al estudio de la cortesía del español peninsular resulta improcedente dada la realidad social española, según ha sido comentada por intelectuales y filósofos. En consecuencia, se insiste aquí en la necesidad de entender la cortesía como un fenómeno social (además de meramente lingüístico), como ya hiciese Fraser (2002), para quien los paradigmas que sirven de raseros a los estudios de cortesía, especialmente el propuesto por Brown y Levinson (1987), adolecen de un sinnúmero de imprecisiones que dificultan todo cometido científico. A partir de la bibliografía con que actualmente contamos, se argüirá que las características de la cortesía española no sólo difieren considerablemente de las de la británica, sino así también de las de otras lenguas germánicas y románicas, como el danés y el portugués. Finalmente, se abogará por la conveniencia de aplicar la social norm view (o norma social) propuesta por Fraser (1990) a los estudios de cortesía española, poniéndose como ejemplo el análisis del concepto face en español, acometido muy recientemente en otro lugar (Ardila 2005). Se demuestra así que la normal social resulta una aproximación eficaz para la comprensión de la cortesía hispánica, además de para trascender esos lugares comunes que de un tiempo a esta parte han adquirido carta de naturaleza - -y que quizá deriven del hecho de que, como apunta Iglesias, los estudios de cortesía en español se han atenido fundamentalmente a las teorías de Brown y Levinson, mientras que el influjo de las propuestas de Lakoff, Leech y Fraser "ha sido mucho menor" (2001: 245)-.

\section{La cortesía española en contraste}

Entre los estudios comparatistas que han tratado la cortesía en español e inglés se encuentran los de Walters (1979a, 1979b), Hickey (1991), Vázquez Orta (1995), Hickey y Vázquez Orta (1994), Woodward (1997), Placencia (1992 y 1995), García (1989), Koike (1994), Mir (1992), Romero (1997), Márquez Reiter (1997, 1998 y 2000), Ardila (2001b, 2002, 2003, 2004 y 2005) y Ardila y Neville (2001). Fant (v.g. 1992) y Kjærbeck (2001), entre otros, han comparado las estrategias retóricas del español con las de lenguas escandinavas. Tan copiosa contribución sitúa al español entre las lenguas que más atención han recibido en los estudios de cortesía. Las diferencias advertidas no son pocas. En el caso del español peninsular y el inglés británico, las reflexiones conceden al español una marcada tendencia a la cortesía positiva, que contrasta con la cortesía negativa del inglés de Gran Bretaña'. En uno de los trabajos que mayor repercusión han tenido, el títulado "Politeness as Deference: A Pragmatic View", Hickey y Vázquez Orta (1994) apuntaron las diferencias respecto de la cortesía lingüística que entre las lenguas española e inglesa median. En la opinión de estos autores:

\footnotetext{
1 Para un conocimiento exacto de la cortesía negativa y la cortesía positiva, refiérase el lector a Brown y Levinson (1987), o a los capitulos "La imagen positiva" y "La imagen negativa" en Haverkate (1994: 21-28, 28-38). Janet Holmes sintetiza las caracteristicas de estos dos tipos de cortesía apuntanto que "negative politeness strategies will occur more often in formal settings and interactions, while positive politeness tends to characterise more intimate and less formal situations" (1995: 20), por lo que identifica la cortesía negativa como la formal y la positiva como la familiar o informal.
} 
Towards their in-group, Spaniards behave with spontaneity and enthusiasm and tend to express their feelings overtly. Members of the same in-group see it as their duty to help and support each other, both morally and financially, so they find no obvious reason for thanking or apologizing, except for something they conceive of as being very serious or beyond the normal duties of the performer of the action [...] In England, cultural norms demand a more distant system of behaviour [...] requests are comparatively more sparingly employed, since they are perceived to a greater extent as impositions and they are preferably expressed more elaborately and indirectly. Verbalizations of thanks and apologies are imperative even for minor relevant situations and among members belonging to the same in-group (1994: 280-281).

La propuesta de Hickey y Vázquez Orta es un claro apunte de las diferencias entre la cortesía positiva y la cortesía negativa que prevalecen en España y Gran Bretaña, respectivamente. A estos autores han seguido, fundamentalmente, Márquez Reiter (1997) y Ardila (2001b y 2002), quienes coinciden en que las diferencias más evidentes entre la cortesía lingüística en inglés y español vienen impuestas porque la británica es una cultura de cortesía negativa, y la española, de cortesía positiva. En La cortesía verbal, Haverkate (1994) comparó el español con el holandés: "De estos análisis se extraen dos conclusiones, una específica, otra general. La específica es que la cultura española difiere de la holandesa en que, por regla general, no concede valor particular al emitir fórmulas de agradecimiento como respuestas verbales a actos rutinarios efectuados dentro de un patrón interaccional preestablecido" (1994: 95). Esta afirmación es asimismo aplicable al inglés británico.

Al abrigo de estos estudios de tenor eminentemente teórico han surgido otros de carácter empírico que cifran con mayor precisión el alegado desuso de las fórmulas de cortesía en español. En un trabajo muy reciente, Ballesteros Martín (2001) disecciona las exhortaciones impositivas en estas dos lenguas y observa una acusada diferencia entre los usos británicos y los españoles. Luego de señalar que "los españoles muestran una clara preferencia por atenuar la fuerza ilocutiva de sus ruegos y mandatos con estrategias de cortesía positiva $(40 \%)$ y los ingleses con estrategias de cortesía negativa $(73,33 \%)$ " (2001: 191), Ballesteros (con Hickey y Vázquez Orta [1994] como única referencia y obviando el resto de los estudios transculturales en torno a la cortesía en inglés y español) elucubra a modo de conclusión:

no se puede catalogar una sociedad como más cortés que la otra, sino que se trata de dos tendencias divergentes a la hora de conceptualizar el fenómeno, que ocasiona que los españoles lo identifiquen en mayor número de ocasiones con mecanismos de acercamiento social, y que los británicos lo hagan en más ocasiones con mecanismos de distanciamiento social (2001: 194).

Sin embargo, la extrema parquedad hispánica en el uso de estrategias de cortesía precisa de matizaciones más concretas que la simple tesis de "dos tendencias divergentes" que esgrime Ballesteros (supra): sería conveniente precisar la naturaleza de esas "tendencias divergentes" y, por supuesto, las razones que las expliquen. Hasta la fecha, las dos únicas exégesis críticas se deben a Bravo (1999) y Ardila (2005). 


\section{La confianza en la cortesía española}

En un artículo en torno a la dicotomía imagen positiva-imagen negativa, Bravo enumera un total de seis "premisas culturales en España" (1999: 168-169) que condicionan la comunicación lingüística. En la última de estas premisas apunta esta lingüista argentina que "Entre españoles, la relación social se manifiesta a través del establecimiento de grados de 'confianza interpersonal', la cual es entendida en el sentido en que se sabe a qué atenerse con respecto al otro y que se puede hablar sin temor a las ofensas" (Las cursivas son de Bravo, refiriéndose a Thurén), idea que Bravo recoge de Thurén (1988: 222). Seguidamente concreta Bravo:

Los principios que describen este tipo de confianza varían dependiendo de los roles relativos de los participantes en la conversación, por ej [sic]; si se está entre amigos, familiares o compañeros de trabajo, y abarcan una amplísima gama de acepciones que se aplican en diferentes escenarios. En realidad, la confianza interpersonal constituye un ideal de relación interpersonal trasladado de tipos de relaciones como las que se mantienen dentro del grupo familiar o entre amigos a las situaciones cotidianas de conversación (1999: 169-170).

Bravo alcanza esta hipótesis de la confianza a partir de las teorías socioculturales expuestas por Porter (1972), Alwood (1986) y Arnstberg (1988), según las cuales, explica Bravo, "La conciencia de pertenencia a una comunidad incluye sobre todo conceptos acerca del Yo, del universo y de sus relaciones" (1999: 157), que entiende que quedan delimitadas por sendos estudios de Madariaga (1928) y Crespo (1986).

Una atenta lectura de las seis premisas revela ciertas contradicciones. Procedería apuntar, a modo de ejemplo, que carece de sentido que en la premisa 3 se atribuya al español una "necesidad de autonomía [que] aparece también relacionada con la noción del orgullo del individuo" (1999: 168) y la premisa 5 mantenga que "Existen entre los miembros de esta sociedad una tolerancia hacia la expresión de opiniones aun cuando difieran de las propias" (1999: 169). La tolerancia está reñida con el orgullo, porque el orgullo, como apunta la misma Bravo, "da lugar [...] a comportamientos defensivos y desafiliativos [sic] de [sic] parte del afectado" (1999: 168)2. En lo que atañe a la confianza cumple precisar que, según la define Bravo, debería procurar el aprecio de los interlocutores, con el obvio objeto de facilitar la comunicación. En la premisa 1 establece Bravo que "ser original y consciente de las buenas cualidades propias" es "condición para obtener el aprecio del entorno", pero también da lugar a "conflictos sociales" (1999: 168). Resulta, por ende, muy difícil comprender cómo se puede aplicar la confianza propia de "amigos, familiares o compañeros de trabajo" a "diferentes escenarios" (1999: 169) si los hablantes saben que puede derivar en "conflictos sociales" (supra).

Briz (1999) reconoce la existencia de la confianza en el español coloquial. En un trabajo posterior, Hernández Flores (2001: 89; vid. asimismo Hernández Flores, 2004a, 2004b) describía la confianza en función de cuatro premisas: 1) tener la confianza del interlocutor

2 Siguiendo a Crespo (1986: 216-217), Bravo establece una distinción entre tener orgullo (que es una cualidad loable) y ser orgulloso. Toda suerte de "comportamientos defensivos y desafiliativos" (Bravo, 1999: 168) deriva de actitudes orgullosas, más que de tener orgullo. 
significa disfrutar de una relación cercana y afectiva con él; 2) valerse de la confianza implica hablar con franqueza y sin reservas; 3) ser una persona de confianza exige ser percibido por el interlocutor como un amigo, casi como un familiar, y 4) actuar asistido por la confianza permite expresarse libremente, sin temor a ofender al interlocutor y a sabiendas de que nuestros actos serán interpretados con naturalidad. Mediante la confianza, argumenta Bravo (1999), los españoles interaccionan verbalmente de modo muy dispar al resto de la civilización occidental. Es la confianza, indica Bravo (sufra), la responsable de que en español se obvien, con mayor o menor asiduidad, las fórmulas de cortesía y se procure dotar todo acto comunicativo de familiaridad.

Los trabajos de Briz (1999) y Hernández Flores (1999, 2001, 2004a) toman como referente el español hablado en un contexto familiar ${ }^{3}$. Por el contrario, estudios más recientes (vid. infra) han aplicado el concepto confianza a la interacción en contextos empresariales. $\mathrm{El}$ análisis más profundo, metódico y, quizá, sugerente de la confianza corresponde a Villemoes (2002, 2003; Villemoes y Kjærbeck, 2003). En el I Symposium on Intercultural, Cognitive and Social Pragmatics de la Universidad de Sevilla, Villemoes presentó una comunicación de título "How Do Spaniards Create the Trust Necessary to Initiate Negotiations with Strangers"4, cuyos argumentos giraban en torno a la confianza, acerca de la cual asevera: "The Southern Spaniard prefers to negotiate creating confianza, which triggers friendship and vice versa". Villemoes establece las siguientes hipótesis:

The Spanish negotiator knows all about confianza and the rules governing its use. The Spanish negotiator supposes that the stranger with whom he is about to negotiate doesn't know the rules. The Spanish negotiator knows that getting to know a stranger is a time consuming and complicated process. He also knows that he will have the upper hand if he succeeds in having his counterpart accept "the confianza game".

Y concluye: "Yes, the Southern Spaniard knows what he is doing. He knows that he is using confianza where it doesn't belong, but he takes the risk that the counterpart will not call his bluff, because he prefers to interact the confianza way, and because the possible gains are immense". En su comunicación, Villemoes adelantaba los argumentos que publicaría inmediatamente después, en un artículo aparecido en la revista Hermes (Villemoes, 2003) y en el libro Negociar a lo español (Villemoes et al., 2004), donde presenta la confianza como un componente endémico a la retórica interpersonal del español. La tesis de la lingüista danesa se apoya en las teorías de Williamson (vid. Villemoes, 2003: 119-120) y Gudykunst (vid. Villemoes, 2003: 119-120), así como en una serie de entrevistas (llevadas a cabo por ella) con profesionales de la negociación, españoles y no españoles (ocho individuos y tres grupos de nueve individuos cada uno, lo que monta un total de 11 horas [i.e. 8 entrevistados $\mathrm{x} 1$ hora +3 grupos $\mathrm{x} 1$ hora]).

En su artículo, Villemoes establece que sus argumentos atañen exclusivamente a las regiones meridionales de España y, dentro de ese límite geográfico, a la negociación en las pymes. Establecidos estos parámetros, Villemoes presenta las siguientes conclusiones: 
1. "las variaciones regionales [...] nos obliga[n] a distinguir por lo menos entre dos formas distintas de negociar, o sea entre la forma de negociar vista en Madrid y en Andalucía, por un lado, y otra vista en Cataluña y el Norte de España" (Villemoes y Kjærbeck, 2003: 57).

2. "The Southern Spaniard in small and medium-sized enterprises when doing business prefers to negotiate in the confianza-mode. This triggers friendship which again calls for informal conversation in informal places in a very individualized way, self-affirmative behaviour, creativity and imagination, flexibility, dynamism, improvisation, and lack of preparation" (2003: 130).

3. "In order to support the confianza the Spaniard turns on his charm, his self-affirmation comes into play, and he focuses his attention one hundred per cent on the interlocutor, all of which is seen by the foreigners as 'the charismatic charm of the Spaniard"" (2003: 129).

4. "quite a few of the characteristics of Spanish negotiation fit nicely into an ambiance of friendship rather than into one of business" (2003: 129).

Por bien argumentadas que estén (y lo están), estas propuestas pueden prestarse a controversia. En primer lugar, es menester reparar en que mientras que, en el artículo publicado en Hermes, Villemoes (2003) traza una linde en el mediodía peninsular y adjudica la confianza a las regiones situadas al sur, en el libro Negociar a lo español Villemoes y Kjærbeck (2003) reconocen su uso en toda España, si bien sitúan un empleo acentuado de la misma en el sur: "Teniendo en cuenta [...] las diferencias regionales, parece que este concepto de la negociación [i.e. la confianza] está más arraigada en el Sur de España" (Villemoes y Kjærbeck 2003: 57). Si no una contradicción, esto bien puede entenderse como una imprecisión. En cualquier caso, el estudio de Pérez Parent (2001), donde se demuestra la ausencia de marcadores de cortesía en actos comunicativos formales en Cataluña, invalida que la confianza sea patrimonio exclusivo del "Southern Spaniard"; es muy probable, también, que analíticas del español hablado por el "Northern Spaniard" pudieran arrojar datos similares a los recogidos por Pérez Parent (2001).

En segundo lugar, Villemoes estima que los españoles gustan de centrar toda su atención en "the interlocutor, all of which is seen by the foreigners as 'the charismatic charm of the Spaniard" (supra), afirmación que quizá contradigan Villemoes y Kjærbeck (2003: 82) al reconocer en el español una acusada individualidad. Aun así, Villemoes adjudica al español un encanto carismático, apreciación esta de didáctica ad usum Delphini y que recuerda estereotipos de otrora o, cuando menos, al que muchas mujeres escandinavas (por poner un ejemplo) tienen de los españoles.

Por último, siguiendo a Bravo (1999), Villemoes observa que las negociaciones que en otras culturas pertenecen a un contexto de formalidad, en España adquieren de ordinario forma de familiaridad y crean un ambiente amistoso. Villemoes y Kjærbeck escriben en este respecto que "el mundo que intentan crear los españoles del Sur y del Centro en la negociación es el mundo del amiguismo" (2003: 57) y que en las pymes del sur "juega un papel la negociación basada en el amiguismo y en la poca preparación, mientras que las empresas grandes, sean nacionales o multinacionales, encarnizan [sic] la preparación y parece que casi no valoran del todo las personas y los eventuales lazos de amistad" (Villemoes y Kjærbeck, 2003: 73). Habría que distinguir entre lo que es y supone esta transposición de 
contextos -i.e. adoptar las estrategias dialécticas propias de un contexto de familiaridad cuando y donde procederían estrategias de formalidad - y lo que es el amiguismo, además de determinar si el amiguismo es exclusivo de la cultura española o se produce igualmente en otras (siquiera fuese en menor medida) como las angloparlantes o las escandinavas.

Por ello, a partir de estas primeras precisiones, las hipótesis y las conclusiones de Villemoes (y Kjærbeck) suscitan no pocas cuestiones. Si todo participante en un proceso de negociación es negociador, no parece muy probable que - siendo el español consciente de los efectos y usos de la confianza- el negociador suponga que "the stranger with whom he is about to negotiate doesn't know the rules" (supra). En efecto, como sugiere Villemoes, el negociador se sabría en clara ventaja si su interlocutor acepta "the confianza game" (supra); así las cosas, es evidente también que tales apreciaciones precisan ser matizadas.

Ha de entenderse, según la exposición de Villemoes, que la confianza tiene por objeto producir un sentir de amistad, y que dicho sentir tiene por razón "to have the upper hand" (supra) en el proceso negociador. Dichas premisas sugieren la siguiente cuestión: en un país en que la corrupción en las más altas instancias fue muy habitual durante la primera mitad de los años 90 (y que ya en el siglo XVII acabó con la privanza del Duque de Lerma [cf. Feros, 2000], quien, por cierto, por ser de Valladolid era del norte), donde, según denuncian algunos intelectuales, el engaño puede llegar, a ser moneda de cambio (cf. Pérez-Reverte 2003), ¿quién se va a fiar de nadie por mor de esa confianza que sirve al negociador para "to have the upper hand"? La realidad social española que describe (v.g:) Pérez-Reverte (vid. infra) insta a colegir que lo único que puede connotar confianza en una negociación ha de ser la adopción de las formas propias de un contexto de formalidad, que muestre al interlocutor la seriedad y la profesionalidad que verdaderamente merecen confianza.

La confianza asoma en todo tipo de instituciones (cf. Ardila, 2005), si bien se prodiga en bares, kioscos y otros pequeños establecimientos; sin embargo, es muy poco común, verbigracia, en El Corte Inglés, que quizá sea la empresa española que cuenta con la mayor confianza del cliente. Un ejemplo muy reciente de empresa española de gran prestigio que ha mostrado su abierto repudio por toda forma de confianza es Iberia, que en enero de 2004 impuso a sus empleados el uso de una Guia de estilo propia, en que se prescribe el trato que a los clientes se debe y que se rige por todo aquello que es contrario a la confianza. En pura lógica, la confianza habría de ser endémica a aquellas empresas que cuentan con la confianza del cliente; mas no lo es, como demuestran El Corte Inglếs e Iberia. Muy por el contrario, no es difícil hallar ejemplos de quienes perciben el exceso de confianza hispánico como una mera falta de decoro social. En un artículo reciente, Arturo Pérez-Reverte (2003) relata sus vivencias como cliente en ocasiones en que los vendedores se armaron de confianza y le propusieron efectuar el pago en dinero $B$. Pérez-Reverte critica adustamente la naturalidad (término que emplea Hernández Flores [2001: 89]) con que los vendedores le proponían defraudar al fisco: "Lo malo es cuando todo se vuelve tan natural y público que perdemos la vergüenza", tan natural que, continúa el académico, "En un país donde el dinero negro se mueve con naturalidad impúdica, desde la princesa altiva a la que pesca en ruin barca, desde el fontanero que pregunta si lo quieres con o sin factura hasta el que te vende el coche y la casa, ocho de cada diez fulanos apuntan una sugerencia más o menos explícita" (2003: 10). Las dos frases que cierran el artículo explican la opinión que a PérezReverte merece la confianza que los vendedores le mostraron: "Y eso fue lo que más me fastidió: el compadreo" (2003: 10). 
Efectivamente, en España es habitual esa confianza, o el exceso de ella, en contextos formales; ahora bien, que la confianza sea aceptada por los españoles, ya sean sureños o norteños, es algo que ponen en tela de juicio las empresas más importantes, como El Corte Inglés e Iberia, y, al menos, uno de los intelectuales más conocidos y reputados de la España de entre siglos. Por todo lo anterior, es necesario rechazar el término lingüístico confianza en los términos en que se ha presentado, ya que esta confianza acaso no haga más que suscitar, en la mayoría de las instancias, la desconfianza del interlocutor — con la obvia salvedad de ciertos contextos situacionales de familiaridad, como los analizados por Briz (1999) y Hernández Flores (2001) - . Todo esto lo advirtió hace varias décadas Dámaso Alonso, quien opinaba que el uso excesivo de tú (en lugar de usted), por mor de lo que después se ha denominado confianza, ha derivado en una suerte de afasia semántica, donde el tuteo ha perdido su valor anterior de verdadera confianza: "iQué suave era el usted, qué sincero, cuántos matices permitía! La amistad, el tú, se ganaban, se construían lentamente. El tú era entonces un verdadero tu'" (1962: 266), lamentaba Alonso.

\section{La pragmalingüistica del español en contraposición al inglés, el danés y el portugués}

En otro lugar (Ardila 2001a), y divagando en torno a la negociación transcultural, se hacía referencia a un estudio de Cary Cooper (citado en Simpson, 1998: 36) donde se exponían las preferencias de ejecutivos en sus negociaciones internacionales: "Americans prefer the British; the British prefer Americans; Danes prefer the British; the Dutch prefer Americans and Germans; the French prefer the French; the Germans prefer the Germans; and Swedes prefer Americans" (Ardila, 2001a: 337). Para justificar sus predilecciones, los americanos, los suecos y los holandeses valoraron la profesionalidad, y los británicos y los daneses, la actitud positiva (que no la cortesía positiva) de los interlocutores. Cabe preguntarse qué valor atesora la confianza hispánica en cuadros de esta índole. En noviembre de 1992 el rotativo The European publicó los resultados de una encuesta en que requería a 600 ejecutivos europeos a que valorasen ciertas características culturales en ocho nacionalidades conforme a la imagen que los encuestados tenían de los nacionales (reproducido en Gibson, 2002: 85). Tras los italianos (198 puntos), los españoles aparecieron como quienes más desconfianza suscitaban (141 puntos) -frente a los holandeses (47 puntos), los japoneses (63 puntos), los alemanes (71 puntos), los norteamericanos (94 puntos), los británicos ( 94 puntos) y los franceses ( 94 puntos)-. Los españoles quedaron a la zaga del resto de nacionalidades en los apartados de éxito profesional (con 16 puntos frente a los 199 de japoneses y alemanes), ambición (27 puntos frente a los 201 de alemanes) y esfuerzo en el trabajo (con 38 puntos frente a los 248 de los japoneses), siempre muy por debajo de las valoraciones recibidas por otras culturas. Estos tres son atributos que condicionan la confianza que se pueda tener en un colectivo o en un interlocutor, y aunque la percepción que los españoles suscitan haya podido cambiar significativamente desde 1992 —por efecto de la buena imagen dada en las olimpiadas de Barcelona y de los notables logros en materia económica - no es arriesgado convenir en que aún inspiran más desconfianza que otras muchas nacionalidades.

De los argumentos de Villemoes se infiere que la confianza debiera hacer que británicos y daneses prefirieran al español, por cuanto esa confianza, a decir de Bravo y Villemoes (supra), rezuma una actitud positiva; sin embargo, no existen evidencias de ello. En el 
ámbito de la sociopragmática (tal como la concibe Leech ${ }^{5}$ ), la confianza nos obliga a traer a colación el parecer de Haverkate (cf. Stewart, 1999: 178, para el caso del inglés), para quien:

La conclusión general es que las diferencias prescritas pueden dar lugar a malentendidos o conflictos de tipo etnolingüístico. Así pues, no es raro que, dentro del contexto de los actos rutinarios, el comportamiento interaccional de los españoles les parezca descortés a los holandeses, mientras que a los españoles la reacción verbal preferida por la cultura holandesa les dé la impresión de ser exagerada o superflua (1994: 95).

El cotejo del español y su supuesta confianza con cualesquiera de las lenguas a que se refiere Cooper arrojaría un sinnúmero de estridentes divergencias entre los modelos de cortesía, pero, como sugiere Haverkate (supra), sería fuente inagotable de errores sociopragmáticos y pragmalingüísticos. Por ello, más que reducir la cuestión a la existencia de unas "tendencias divergentes" (Ballesteros supra) o de la confianza lingüística y sus románticas exégesis (Villemoes supra), es hacedero abordar lo único que se ha probado empíricamente: el desuso en español de las fórmulas y las estrategias de cortesía que en otras lenguas son condición sine qua non para evitar la fricción en la comunicación (que es una de las definiciones más recurrentes de la cortesía [Chilton, 1990; Ide, 1993; Márquez Reiter, 1997]). Y este rasgo imprime a la lengua española un empaque muy propio. Tómese como ejemplo un contexto familiar, donde en español un hablante dice a su interlocutor:

"Dime qué quieres" y

"Vámonos ya",

el británico (que tanto valora las actitudes positivas) muy bien podría inquirir:

"Can you tell me what you want?" y

"Should we go now?",

y el danés (ávido también de mostrar y recibir una actitud positiva):

"Kan du ikke sige mig, hvad du ønsker?" y

"Skal vi gå?"

Dentro de las fronteras de las lenguas romances, el portugués seguramente emplease las fórmulas:

"O qué é que deseja?" y

"Então, vamos embora?"

Evidentemente, los anteriores ejemplos reclaman un soporte empírico que les confiera su justa precisión; sin embargo, en estos, como en los que aparecerán a continuación, me baso

5 "Socio-pragmatics would involve the assignment of variant values", fundamentalmente en diferentes lenguas (Leech, 1983: 18). 
en mi observación indiscriminada de estas cuatro lenguas, como ya hicieran Haverkate 1994) o Ardila (2002, 2003), y en el caso del inglés, por ejemplo, Thomas (1995).

A Márquez Reiter (1997; vide asimismo Márquez Reiter, 1998, 2000) se debe una de las sistematizaciones más completas de las discrepancias pragmáticas entre las lenguas española e inglesa. Tomando los datos de un estudio empírico anterior, Márquez Reiter señala que "Both in Spanish and English requests can be linguistically realised with imperatives, interrogatives, negative interrogatives and declaratives" (1997: 145); no obstante, la recurrencia de cada una de estas estrategias variará considerablemente de una lengua a otra. Las investigaciones de Márquez Reiter sobre peticiones indican que en español el uso de imperativos es significativamente más recurrido que en inglés (un 29 por ciento frente a un 10 en su análisis), y matiza: "In Spanish, however, imperatives are not simply used for commands and instructions, they are also used to express hopes, desires and wishes, and they are much more frequent than in English" (1997: 146). En cuanto a las peticiones interrogativas, el inglés registró un 86 frente al 68 por ciento del español, por lo que, sugiere la autora, "In English there appears to be a stronger preference for more elaborate constructions with modals whereas in Spanish they are generally formulated with the present indicative or conditional constructions" (1997: 146). Las frases declarativas son también más recurrentes en inglés que en español y las peticiones que en español se valen de los verbos necesitar y querer "may sound too direct and impolite to English speakers" (1997: 147), toda vez que el inglés prefiere las declarativas.

La cuestión se reduce, pues, a que el español se rige por una cortesía positiva mientras que el inglés mantiene un modelo de cortesía negativa. Por ello, Márquez Reiter concluye:

It is probably due to the differences in the values attached to positive and negative politeness that the British regard the Spaniards as flatterers and bad-mannered and the Spaniards regard the British as unfeeling and hypocritical. The whole point is that what constitutes politeness may differ from one culture from another and thus it can be realised linguistically in different ways (1997: 145).

Aun cuando los valores sociales no han de coincidir en culturas diferentes ${ }^{6}$, Brown y Levinson no titubearon al aseverar que la cortesía negativa es "the heart of respect behaviour" (1987: 129). Márquez Reiter (supra) ha sabido mostrarnos en qué medida la cultura británica se vale más de lá cortesía negativa que la española, y lo cierto es que análisis comparatistas de similar índole podrían demostrar con cifras que lenguas germánicas como el danés operan con modelos de cortesía muy distintos al español, lo que se evidencia, verbigracia, en las peticiones, que Márquez Reiter ha analizado en inglés.

En danés, el imperativo es empleado en un porcentaje significativamente inferior al español, y mientras que el español, en contextos formales, muy bien puede enunciar "Ven aquí", "Dime" y "No te olvides", imposiciones del tipo "Kom her", "Sig mig" y "Glem det" son irrecurrentes en danés en un contexto situacional similar (adviértase, no obstante, que la forma "Kom lige her" sería más cortés). Asimismo, el uso de interrogativas excede al español, y donde el danés diría "Kan du komme her?", "Kan du sige mig?" o "Vil du glemme det?",

6 Cf. Georgia Green: "Obviously, cultures will differ in terms of what counts as conventionally polite in various stereotypical situations" (1989: 145). 
el español enunciaría "Ven aquí", "Dime" y "No te olvides". En un contexto profesional, la enunciación castellana "Dime" (en inglés "How can I help you?") se expresaría en danés "Hvad kan jeg hjælpe dig med?". En danés, además, el uso de las interrogativas negativas es habitual con el verbo Kan, y los ejemplos apuntados tendrían en "Kan du ikke komme her?" y "Kan du ikke sige mig?" sus variantes más recurridas, especialmente en contextos de formalidad y familiaridad. Por último, las frases declarativas, tan irrecurrentes en español, son ciertamente habituales en danés: mientras que el español enuncia "El periódico" o "Un café" (como peticiones), el danés se expresaría por medio de las declarativas "Jeg vil gerne have en kop kaffe" y "Jeg vil gerne have avisen". Además de los usos en las peticiones, es notorio que en danés el empleo de los marcadores de cortesía Vaer så venlig, Venligst, Undskyld, Tak o Mange tak es muy superior al uso de Por favor y Gracias (casi inexistente en España, según Pérez Parent y. Hernández Flores supra).

Si llevamos nuestro cotejo a latitudes latinas, repararemos asimismo en que la cortesía positiva del español no halla correspondencia entre sus hermanas romances. El portugués es un ejemplo de lengua romance que se aferra a un estricto modelo de cortesía negativa. En otro lugar (Ardila, 2005) se empleó el portugués para destacar la cortesía positiva del español. Allí se reflexionó en torno a las formas de tratamiento, en portugués tu, vocé y o senhor / a senhora, y en cómo el uso de las mismas no se corresponde con el uso español en el caso de los contextos situacionales de formalidad y familiaridad. Aun cuando el portugués no es remiso al uso de imperativos, estos suelen acompañarse de marcadores de cortesía; así, los imperativos españoles "Ven aquí", "Dime" y "No te olvides" tendrían sus equivalentes portugueses en "Faça favor de vir cá" o "Venha cá, faça favor", "Faça favor de dizer-me" y "Faça favor de não esqueçer". En contextos profesionales, el "Dime" español hallaría su equivalente portugués en "Faça favor". El portugués preferiría asimismo las construcciones interrogativas, i.e. "Pode vir cá?", "Pode dizer-me?" y "Poderá lembrar-se?". A pesar de que las interrogativas negativas y las declarativas tengan una mínima incidencia en portugués, como en danés, los marcadores de cortesía en esta lengua, Faça favor, Obrigado u Obrigada (y Obrigadinho u Obrigadinha), Com licença y Disculpe, se prodigan con superior asiduidad que en español.

\section{La norma social en los estudios de cortesía}

Como apuntan los ejemplos anteriores la cortesía positiva española — como puede observarse en el uso y el abuso de la confianza - diverge de los modelos de cortesía negativa de otras lenguas de su entorno cultural, tal que el inglés y el danés - que, en contextos profesionales, valoran actitudes positivas (que no cortesía positiva) - y el portugués, lo que suscita e invita a considerar las razones de dicha impronta. Esta cuestión se presta a dos reflexiones que han sido evitadas (o acaso ignoradas) por los estudios de cortesía: primero, si las posturas positivas y la ausencia de fórmulas de cortesía son, como sugiere Villemoes, características de ciertos estratos; $y$, segundo, qué razones pueden explicar o justificar estos usos.

Nos llevaremos una somera sorpresa si exponemos los pareceres de una serie de intelectuales españoles de renombre frente a la prudencia de aquellos lingüistas que proclaman que ninguna cultura ha de ser tenida por más o menos cortés que cualesquiera otras. Las letras españolas son prolijas en admoniciones contra una supuesta descortesía hispánica, desde 
Gracián en el Criticón a Larra en sus Artículos de costumbres. En el siglo XX, Ortega y Gasset, el filósofo europeo más influyente de su época, tildaba a España de "imperio indiviso de la descortesía" (1969: 67) y ya en el siglo XXI Javier Marías ha aseverado que "En España no existe la cortesía apenas, desde hace tiempo, y cuando la hay, aparente, es para incurrir en mayores zafiedad y grosería" (2002: 8). Es decir, a guisa de paréntesis, que mientras que reflexiones en torno a la (en la terminología de Watts, Ide y Ehlich [1992]) cortesía española de primer orden la tachan de descortés, los argumentos de los lingüistas prefieren operar con eufemismos como la confianza ${ }^{7}$.

Parece posible, pues, que la cortesía positiva española posea unas peculiaridades que la aproximan a una suerte de desuso de cualesquiera fórmulas de cortesía, sean del tipo de cortesía positiva o negativa. Así lo ha entendido Haverkate, quien no duda en aseverar: "Podríamos decir, pues, que el español adopta una actitud esencialmente racional ante el acto rutinario, inclinándose a ahorrar energía verbal, en tanto que el holandés tiende a acentuar el aspecto social de la interacción, prefiriendo mostrar cortesía positiva" (1994: 95). Ello es apreciable, por ejemplo, en las formas de tratamiento: la inmensa mayoría de los manuales de buenas maneras (Amable, 1989; Miguel, 1991) y los trabajos de investigación (Alonso, 1962; Lorenzo, 1989; Alba et al., 1980; Marín, 1972; Ardila, 2003, 2005) aconsejan el uso de usted y cuestionan la conveniencia del tuteo indiscriminado que es habitual en la sociedad española. Se precisan estudios en este respecto, estudios que enuncien juicios más comprometidos respecto de la cortesía en España, que se atrevan a emplear el término descortesía o, como sugiere Eelen (2001), a concretar la variación del uso lingüístico en el continuo cortesía-descortesía — de modo análogo a cómo Janet Holmes ha hecho en el discurso de las mujeres para aseverar que "Women tend to be more polite than men" (1995: 6; cf. Holmes, 1989, 1990, 1993, 1997; Gordon, 1997; Brown, 1976, 1980)--.. Por el momento debemos considerar que, en España, ese exceso de confianza y esa desviación de los valores han indignado, por ejemplo, a Ortega, Pérez-Reverte y Marías (supra), para quienes la confian$z a$ no sería más que un eufemismo con que se adorna aquello que Pérez-Reverte tilda sin ambages de compadreo (supra) - y se encubren tendencias a la descortesía.

Sabemos que el discurso del hablante español propende hacia la cortesía positiva; sin embargo, los paradigmas con que en la actualidad cuentan los estudios de cortesía nos privan de las teorías que puedan explicar el porqué la cultura española adopta una cortesía positiva mientras que otras que le son muy cercanas propenden a la cortesía negativa. Fraser (2002) reclamó, en el mismo I Symposium on Intercultural, Cognitive and Social Pragmatics y en su ponencia de título "Wither Politeness?", nuevos paradigmas que posibiliten el estudio de la cortesía allende los manidos parámetros de Brown y Levinson. En el caso que nos ocupa, el más apropiado de los moldes analíticos pudiera ser el propuesto por el mismo Fraser en su "Perspectives on Politeness" (1990), cuyos contenidos se adelantaron en "The Association of Difference with Linguistic Form" (Fraser y Nolen, 1981). Fraser (1990) contempla la cortesía desde cuatro views, o perspectivas: la social norm view, que promulga la incidencia de factores históricos y sociales en la cortesía; la conversational maxim view, o máxima conversacional, que se corresponde con el principio de cortesía de Leech (1983) y

7 Con la excepción de Iglesias, quien plantea: "La pregunta que surge en relación con la cortesía es la siguiente: ¿es el español actual menos cortés que el de épocas anteriores?" (2001: 260). Iglesias estima que el español actual es menos cortés si se considera que las fórmulas de cortesía están en claro desuso. 
sus máximas; la face-management view, o dominio de la face, basada en las estrategias que Brown y Levinson (1987) señalan en la conversación para organizar los face-threateningacts, y la conversational contract view o contrato conversacional, que Fraser añade a los anteriores.

Las alusiones a los valores sociales constituyen la primera de las cuatro perspectivas que Fraser confiere al fenómeno comunicativo de la cortesía. Por norma social Fraser significa la concretización de la cortesía a lo largo de la historia de cada una de las culturas, entendiendo las variaciones transculturales como resultado de los diferentes avatares sociales que cada nación ha experimentado. Los valores sociales también han sido considerados por Patricia Clancy (1986), Shoshana Blum-Kulka (1990), Catherine Snow, Rivka Perlmann, Jean Gleason y Nahid Hooshyar (1990), Richard Janney y Horst Arndt (1993) y Henk Haverkate (1994), y Werkhofer no ha vacilado en vindicar los valores sociales como reguladores de la cortesía, arguyendo que la cortesía supone "The power of a symbolic medium that, being used and shaped in acts of individual speakers, also represents social standards of how to behave or what kind of conduct is considered 'just and right'" (1992: 156). En función de estos antecedentes, en otro lugar (Ardila, 2005) se acometió el análisis de la cortesía española desde la perspectiva de la normal social. La consecuencia directa e inmediata fue la equiparación del concepto lingüístico face al concepto cultural español honra.

\section{Face y honra}

La norma social de Fraser facilita la comprensión de un concepto clave en la formulación de las tesis de la cortesía positiva y la confianza: el concepto face, que en cualquier caso depende de los valores axiológicos y etológicos de una sociedad. Al tratar las divergencias entre las formas de tratamiento inglesas y españolas (apud Ardila, 2003), se señalaron dos suertes de causas para tales discrepancias: unas exógenas y otras endógenas. Entre las exógenas se contaba el proceso de linguistic egalitarism (que pudiera verterse como egalitarismo lingiǘstico) experimentado en todos los países occidentales, de que era hija la corrección política; entre las endógenas, las convulsiones sociales y políticas experimentadas por la España del XX (dos dictaduras, una cruenta guerra civil, además de la agitación social de los últimos años de la Restauración), convulsiones que no se han vivido en, v.g., Gran Bretaña o Dinamarca. La esencia de los usos de cortesía española se halla en los factores endógenos no del siglo XX, sino del decurso de la dilatada historia de España. La primera rémora que ralentiza la comprensión de la cortesía española es la incomprensión del concepto face, calcado al español como imagen (v.g. en Haverkate, 1994; Gómez Morón, 1999; Saz, 2000, inter alia) ${ }^{9}$ o fisonomía (Ardila, 2001b), cuando existe una voz española que es (groso modo) significante del significado de face, como se demostró ampliamente en Ardila (2005).

8 Parecer a que apostilla Márquez Reiter la siguiente definición: "Politeness is thus a form of social interaction, a form that mediates between the individual and the social. The polite or impolite act is performed by an individual whose choices for the instrumentality of such an act are based upon collective norms and whose motivation in performing the act is that of structuring social interaction" (2000: 3 ).

9 Según Bravo: "La traducción de face como 'imagen' proviene del curso de Lingüística general, dictado por la Prof. Dra. Beatriz Lavandera en la UBA (Universidad de Buenos Aires), en 1985" (1999: 155). 
Esta relación entre face y honor se vislumbra en el artículo de David Ho (1976) de título "On the Concept of Face", donde se señalaba que la voz face es calco de miànzi y liân, que en mandarín son significantes del significado honor. Basándose en estos presupuestos, Lu Ming Mao (1994) observó la relevancia de face y la desatención que había sufrido en los estudios de Brown y Levinson. Argumenta Mao que face es honor, y que el valor que este concepto atesora varía ostensiblemente entre culturas. El honor es una de las premisas culturales (la cuarta) que Bravo observa en los españoles, por cuando "El honor se pone en juego en una suerte de imagen pública que requiere de esfuerzos para ser consecuente con cualidades, responsabilidades y derechos que la sociedad atribuye a quienes desempeñan roles sociales" (1999: 169). En la obra de Goffman hallamos la descripción más precisa de face:

[Face is] The positive social value a person effectively claims for himself by the line others assume he has taken during a particular contact. Face is an image of self - delineated in terms of approved social tributes-- albeit an image that others may share, as when a person makes a good showing for his profession or religion by making a good showing for himself (1967: 5).

En palabras de Brown y Levinson, face es "something that is emotionally invested, and that can be lost, maintained or enhanced, and must be constantly attended to in interaction" (1978: 66). Face, por consiguiente, no es honor, sino la imagen que "a person claims for himself" y que "others assume", esto es, que face es la imagen de un individuo a ojos de la sociedad, y de la sociedad depende esa imagen. Face diferiría, pues, de honor en que face depende de la opinión que la sociedad posea del individuo, mientras que el honor no depende de la sociedad sino del individuo. Es esta, stricto sensu, la diferencia que en español se expresa mediante las voces honor y honra (cf. Ardila, 2005).

Es decir, que el honor se posee de facto, y la honra, de iure, puesto que la honra es la imagen o fisonomía que la sociedad quiera tener de un individuo. Además de lo expuesto en Ardila (2005), puede notarse también que el español no sólo posee la voz honra para expresar face: el verbo fazferir (también hazferir, o la más moderna zaherir), cuya acepción es (según el $D R A E$ ) "Echar en rostro a uno una acusación o un cargo, hiriéndole con él como si fuese con una cosa material", serviría para designar la acción de face-threat, si en lugar de acusaciones o cargos se produjesen enunciaciones de cualquier índole. En el acto de fazferir, que es faciem ferire, lo que se hiere o amenaza es la faciem, en inglés face, que en español es honra. Todo ello ha sido estudiado en Ardila (2005) siguiendo las pautas de la norma social de Fraser, en Ardila (2005).

\section{Conclusiones}

Como hemos visto, diversos estudios teóricos y empíricos (vid. supra) han demostrado que la cortesía española es del tipo positivo. Junto a esos trabajos lingüísticos, otros (vid. supra) han constatado en el español actual de la península un claro desuso de fórmulas y marcadores de cortesía habituales en otros países europeos así como en el español de hace unas décadas. Parece conveniente para los estudios de cortesía española indagar en las razones por las cuales la cortesía en España es del tipo positivo y por qué y en qué medida 
el uso de fórmulas y marcadores de cortesía se halla en franco declive. A Bravo (supra) corresponde el primer intento por entender el desuso de las convenciones lingüísticas en España. Bravo sugiere que la relajación de lo cortés se debe al afán español por mostrar y ganarse la confianza del interlocutor, hipótesis que han desarrollado después varios lingüistas. Aun cuando esa pretensión de confianza puede percibirse, en espacios familiares, como una práctica compatible con la cortesía, en este artículo se ha tratado de demostrar que su empleo en contextos formales resulta tan ilógico como inapropiado y que la misma formulación del concepto confianza en estudios de cortesía peninsular (en contextos formales, insisto) resulta inaceptable a la luz de las políticas de ciertas empresas. La aplicación del término confianza a contextos formales es más una justificación de ciertos usos descorteses que una explicación de los mismos.

Así las cosas, en España, la confianza - según la han descrito lingüistas como Bravo y Villemoes - asoma asiduamente en contextos formales, donde es antagónica y nociva para la cortesía. Este fenómeno no se produce en otros países europeos, tanto en lenguas germánicas (tal que el inglés y el danés) como en otras romances (v.g. el portugués). Los trabajos empíricos publicados en el área de los estudios interculturales demuestran que, a nivel transnacional, los españoles se encuentran entre los europeos que menos confianza inspiran en el extranjero. Toda vez que las teorías de Bravo y Villemoes faltan tanto a la lógica como a la realidad de la sociedad española y de los estudios interculturales, debemos alcanzar a entender los usos lingüísticos peninsulares por otros caminos. En este sentido, resulta especialmente provechoso e interesante tratar de aplicar la norma social de Fraser al estudio de la cortesía en español. Tras Bravo y sus seguidores, el primer intento lo hallamos en Ardila (2005), donde se aborda la cortesía española entendiendo por el concepto face la honra de modo que se expliquen los usos lingüísticos.

Luego de contender la validez de la confianza, en este artículo se ha reseñado y simplificado la correlación entre face y honra y, a los argumentos dados en Ardila (2005) se ha aportado el paralelismo entre el término lingüístico face-threatening y el verbo español fazferir. De las tesis en torno a la honra contenidas en Ardila (2005) y de la refutación de la confianza que aquí se ha llevado a cabo se sigue la necesidad y la conveniencia de que los estudios de cortesía peninsular trasciendan los límites de la pragmática y se adentren en el campo de los estudios sociales y culturales - lo que han aconsejado, en el ámbito de los estudios de cortesía, Fraser (1990) e Eelen (2001). De hecho, la preocupación por la honra se he revelado imprescindible en ciertos estudios literarios y sociales. Basta con releer los textos más celebrados de la literatura áurea para reparar en cómo la honra dominaba la sociedad, como por ejemplo en El alcalde de Zalamea, el Lazarillo, el Guzmán de Alfarache o el Buscón, lo que han denunciado, entre otros, Américo Castro (1966) y Alan Francis (1978). Sin remontarse tantos siglos, Haverkate (1994) halló en el ensayo El español y los siete pecados capitales de Fernando Díaz-Plaja las exégesis de sus observaciones pragmalingüisticas y Bravo (1999) siguió las observaciones de Salvador de Madariaga, lo que demuestra la utilidad de la norma social.

\section{Referencias bibliográficas}

Alba de Diego, V. y J. Sánchez Lobato (1980): "Tratamientos de juventud en la lengua hablada. Aspectos sociolingüísticos", Boletín de la Real Academia Española, págs. 95-129. 
Alonso, D. (1962): "La muerte de usted". En Del siglo de oro a este siglo de siglas. Madrid, Gredos, págs, 264-267.

Alwood, J. (1986): “Mentalitet och sprăk: några reflektioner”. En Sällström, P. (ed.): Mentaliteter. Åbo, Academia de Ábo.

Amable, A. (1989): Manual de buenas maneras. Madrid, Pirámide.

Ardila, J.A.G. (200la): "An Assessment of Cross-cultural Demands within Present-day Foreign Language Teaching", IRAL, XXXIX, 4, págs. 333-339.

- (2001b): "Entornos sociolingüísticos, paralingüísticos y semiológicos de la cortesía: propuesta de una teoría de círculos concéntricos - y sus implicaciones en inglés-español-_". En Moreno A. et al. (dirs.): Perspectivas recientes sobre el discurso. León, Asociación Española de Lingüística Aplicada y Universidad de León, págs. 183-184.

- (2002): "Percepciones semiológicas de los modelos de cortesía británico y español", Estudios Humanísticos. Filologia, XXIV, págs. 229-242.

-- (2003): "(Non-deictic, Socio-expressive) T-/V-pronoun Distinction in Spanish- English Formal Locutionary Acts", Forum for Modern Language Studies, XXXIX, 1, págs. 1-13.

- (2004): "Transition Relevante Places and Overlapping in (Spanish-English) Conversational Etiquette", Modern Language Review, IC, 3, págs. 635-650.

- (2005): Sociopragmática y retórica interpersonal: la cortesia en inglés y castellano. Queenston y Lampeter, Edwin Mellen Press.

Anrstberg, K. O. (1988): "Kultur and Kulturvariation". En Daun, $\AA$ y B. Ehn (dirs.): Bland Sverige. Malmö, Carlssons Bokförlag, págs. 71-87.

Ballesteros Martín, F. J. (2001): "La cortesía española frente a la cortesía inglesa. Estudio pragmalinguístico de las exhortaciones impositivas", Estudios Ingleses de la Universidad Complutense, IX, págs. 171-208.

Blum-Kulka, S. (1990): "You Don't Touch Lettuce with Your Fingers. Parental Politeness in Family Discourse", Journal of Pragmatics, XIV, págs. 259-288.

Bravo, D. (1999): “'Imagen positiva vs. Imagen negativa?: pragmática socio-cultural y componentes de 'face'", Oralia, II, págs. 155-184.

Brown, P. (1976): "Women and Politeness: A New Perspective on Language and Society. Review of 'Language and Woman's Place' by R. Lakoff", Reviews in Anthropology, III, págs. 240-249.

- (1980): "How and Why Are Women More Polite: Some Evidence from a Mayan Community". En McConnel-Ginet S., Borker, R. y N. Furman (dirs.): Women and Language in Literature and Society. Londres, Praeger.

- y S. Levinson (1978): “Universals in Language Usage: Politeness Phenomena”. En Goody E. N. (dir.): Questions and Politeness. Strategies in Social Interaction. Cambridge, Cambridge University Press.

- y S. Levinson (1987). Politeness: Some Universals in Language Use. Cambridge, Cambridge University Press.

Carreira, M. H. A. (2005): "How to Address Others". En Hickey, L. y M. Stewart (dirs.): Politeness in Europe, Londres: Multilingual Matters.

Castro, A. (1966): La realidad histórica de España. Madrid.

Clancy, P. (1986): "The Acquisition of Communicative Style in Japanese". En Schieffelin B. y E. Ochs (dirs.): Language Socialization Across Cultures. Cambridge, Cambridge University Press, págs. 213-249.

Chilton, P. (1990): "Politeness, Politics and Diplomacy", Discourse and Society, 1, págs. 201-224.

Crespo, E. (1986): "A Regional Variation: Emotions in Spain". En Harré, R. (ed.): The Social Construction of Emotions. Londres: Blackwell, págs. 209-217.

Eelen, G. (2001): A Critique of Politeness Theories. Manchester, St. Jerome Publishing.

Fant, L. (1992): "Scandinavian and Spaniards in Negotiation". En Sjögren, A. y L. Janson (dirs.), Culture and Management in the Field of Ethnology and Business Administration. Estocolmo, Stockholm School of Economics. 
Feros, A. (2000): Kingship and Favoritism in the Spain of Philip III, 1598-1621. Cambridge, Cambridge University Press.

Francis, A. (1978): Picaresca, decadencia, historia. Madrid, Gredos.

Fraser, B. (1990): “Perspectives on Politeness", Joumal of Pragmatics, XIV, 2, págs. 219-236.

- (2002): "Wither Politeness?", Conferencia plenaria impartida en el I Symposium on Intercultural, Cognitive and Social Pragmatics. Sevilla, Universidad de Sevilla.

- y W. Nolen (1981): "The Association of Difference with Linguistic Form", International Journal of the Sociology of Language, XXVII, págs. 93-109.

Fredsted, E. (2005): "Getting to the Point". En Hickey, L. y M. Stewart (dirs.): Politeness in Europe. Londres, Multilingual Matters.

Garcia, C. (1989): “Disagreeing and Requesting by Americans and Venezuelans", Linguistics and Education, I, págs. 299-322.

Gibson, R. (2002): Intercultural Business Communication. Oxford, Oxford University Press.

Goffman, E. (1967): Interaction Ritual: Essays on Face-to-Face Behavior. Nueva York, Garden City.

Gómez Morón, R. (1999): "El modelo de cortesía lingüística de Brown y Levinson en los artículos científicos: breve revisión crítica". En Lario, M.C. et al. (dirs.): Enfoques teóricos y prácticos de las lenguas aplicadas a las ciencias y las tecnologias. Cádiz, Universidad de Cádiz, págs. 191195.

Gordon, E. (1997): "Sex, Speech, and Stereotypes: Why Women Use Prestige Forms More than Men", Language in Society, XXVI, 1, págs. 47-63.

Green, G. M. (1989): Pragmatics and Natural Language Understanding. Hillsdale, N.J., Lawrence Erlbaum Associates.

Haverkate, H. (1994): La cortesia verbal: estudio pragmalingüistico. Madrid, Gredos.

Hickey, L. (1991): "Comparative Polite People in Britain and Spain", Association For Contemporary Iberian Studies, IV, págs. 2-7.

- e I. Vázquez Orta (1994): "Politeness as Deference: A Pragmatic View", Pragmalingüística, II, págs. 267-286.

Hernández Flores, N. (1999): "Politeness Ideology in Spanish Colloquial Conversation: The Case of Advice", Pragmatics, IX, 1, págs. 37-49.

- (2001): La cortesía en la conversación española de familiares y amigos. Aalborg, Aalborg Universitet.

- (2004a): "Politeness as 'face' Enhancement: An Analysis of Spanish Conversation between Friends and Family". En Márquez Reiter R. y E. Placencia (dirs.): Current Trends in the Pragmatics of Spanish. Ámsterdam, John Benjamins, págs. 265-285.

- (2004b): "La cortesía como la búsqueda del equilibrio de la imagen social". En Bravo D. y A. Briz (dirs.): Pragmática sociocultural: Estudios sobre el discurso de cortesía en español. Barcelona, Ariel.

Ho, D.Y. (1976): "On the Concept of Face", American Journal of Sociology, LXXXI, págs. 867-884.

Holmes, J. (1989): "Sex Differences and Apologies: One Aspect of Communicative Competence", Applied Linguistics, X, 2, págs. 194-213.

- (1990): "Politeness Strategies in New Zealand Women's Speech". En Bell, A. y J. Holmes (dirs.): New Zealand Ways of Speaking English. Clevendon, Multilingual Matters, págs. 252-276.

- (1993): "New Zealand Women Are Good To Talk To: An Analysis of Politeness Strategies in Interaction", Journal of Pragmatics, XX, págs. 91-116.

- (1995): Women, Men and Politeness. Londres, Longman.

- (1997): "Women, Language and Identity", Journal of Sociolinguistics, I, 2, págs. 195-223.

Ide, S. (1993): "Preface: The Search for Integrated Universals of Linguistic Politeness", Multilingula, XII, 1, págs. 7-11.

Iglesias Recuero, S. (2001): "Los estudios de cortesía en el mundo hispánico. Estado de la cuestión", Oralia, IV, págs. 245-298. 
Janney, R. y H. Arndt (1993): "Universality and Relativity in Cross-cultural Politeness Research: A Historical Perspective", Multilingua, XII, 1, págs. 13-50.

Johnston, B., Ferrara, K. y J. Mattson Bean (1992): "Gender, Politeness, and Discourse Management in Same-sex and Cross-sex Opinion-poll Interviews", Multilingua, XII, 1, págs. 13-50.

Kjærbeck, S. (2001): “'Nosotros los españoles' y 'los de fuera'. Un estudio de focus group sobre la identidad cultural", Discurso y Sociedad, III, págs. 43-74.

Koike, D. (1994): "Negation in Spanish and English Suggestions and Requests: Mitigating Effects?", Journal of Pragmatics, XXI, págs. 513-526.

Lakoff, R. (1973): "The Logic of Politeness: or Minding Your P's and Q's". En Papers from the Ninth Regional Meeting of the Chicago Linguistic Society. Chicago, Chicago Linguistic Society, págs. 292-305.

Leech, G. (1983): Principles in Pragmatics. Londres, Longman.

Lorenzo, E. (1989): "Relación interpersonal y expresión impersonal. En Rodríguez, F. (dir.): Comunicación y lenguaje juvenil. Madrid, Fundamentos, págs. 165-182.

Lozano, I. (1995): Lenguaje femenino, lenguaje masculino. ¿Condiciona nuestro sexo la forma de hablar? Madrid, Minerva.

Madariaga, S. de (1928): Englishmen, Frenchmen and Spaniards. Londres, Oxford University Press.

Marías, J. (2002): "Hacia la ley del más grosero", El Semanal, DCCLXVII, pág. 8.

Marín, D. (1972): "El uso de tú y usted en el español actual", Hispania, LV, págs. 904-908.

Márquez Reiter, R. (1997): "Sensitising Spanish Learners of English to Cultural Differences: The Case of Politeness". En Pütz M. (dir.): The Cultural Context in Foreign Language Teaching. Frankfurt am Main, Peter Lang, págs. 143-155.

- (1998): "The Teaching of Politeness in the Language Classroom". En Vázquez Orta, I. e I. Guillén (dirs.): Perspectivas pragmáticas en lingüística aplicada. Zaragoza, Anubar, págs. 291-297.

- (2000): Linguistic Politeness in Britain and Uruguay. A Constrastive Study of Requests and Apologies. Londres, John Benjamins.

Mao, L.M. (1994): "Beyond Politeness Theory: "Face" Revisited and Renewed", Journal of Pragmatics, XXI, 4, págs. 451-486.

Miguel, A. de (1991): Cien Años de Urbanidad. Barcelona, Planeta.

Mir, M. (1992): "Do We All Apologize the Same? - An Empirical Study on the Act of Apologizing by Spanish Speakers Learning English". En Bouton L. y Y. Kachru (dirs.): Pragmatics and Language Learning. Urbana, University of Illinois at Urbana-Champaign Press, págs. III, 1-19.

Ortega y Gasset, J. (1967): El espectador. Madrid, Salvat.

Pérez Parent, M. (2001): "Some Considerations on Politeness in Catalan Service Encounters". En Muñoz C. (dir.): Trabajos de lingiística aplicada. Barcelona, Univerbook, págs. 597-604.

Pérez-Reverte, A. (2003): "Con o sin factura", El Semanal (28 de septiembre de 2003), pág. 10.

Placencia, M.E. (1992): "Politeness in Ecuadorian and British English", Language Learning Journal, V1, págs. $80-82$.

- (1995): "Explicitness and Ellipsis as Features of Conversational Style in British English and Ecuadorian Spanish", IRAL, XXXIII, 2, págs. 129-141.

Porter, R. E. (1972): “An Overview on Intercultural Communication". En Samovar, L. A. y R. E. Porter (dirs.): Intercultural Communication: A Reader. Belmont, Wasaworth.

Romero, J. (1997): "Your Attention, Please: Pragmatic Mechanisms to Obtain the Addressee's Attention in English and Spanish Conversations", Journal of Pragmatics, XXVIIl, págs. 205-221.

Saz, M.M. del (2000): La cortesía lingïística en el discurso publicitario. Valencia, Universidad de Valencia.

Simpson, L. (1998): "The Culture Trap", Business Life, November 1998, págs. 33-36.

Snow, C., Perlmann, R., Gleason, J. y N. Hooshyar (1990): "Developmental Perspectives on Politeness: Sources of Children's Knowledge”, Journal of Pragmatics, XIV, págs. 289-305.

Stewart, M. (1999): The Spanish Language Today. Londres y Nueva York, Routledge. 
Thurén, B. M. (1988): Left Hand Left Behind: The Changing Gender System of a Barrio in Valencia, Spain. Stockholm, Stockholms Universitet.

Thomas, J. (1995): Meaning in Interaction. Londres, Longman.

Vázquez Orta, 1. (1995): A Contrastive Study of Politeness: Phenomena in England and Spain. Duisburg, LAUD.

Villemoes, A. (2002): "How Do Spaniards Create the Trust Necessary to Initiate Negotiations with Strangers", Comunicación presentada en el I Symposium on Intercultural, Cognitive and Social Pragmatics. Sevilla, Universidad de Sevilla.

- (2003): "How Do Southern Spaniards Create the Conditions Necessary to Initiate Negotiations with Strangers?", Hermes. Journal of Linguistics, XXXI, págs. 119-134.

- y S. Kjærbeck. (2003): "Negociar a lo español". En Villemoes A. et al: Negociar a lo español. Aarhus, Systime Academia, págs. 16-114.

Walters, J. (1979a): "The Perception of Politeness in English and Spanish", TESOL, XXIX, págs. 289-296.

- (1979b): "Strategies for Requesting in Spanish and English - Structural Similarities and Pragmatic Differences", Language Learning, IX, 2, págs. 277-294.

Watts, R., Ide, S. y K. Ehlich. (1992): "Introduction". En Watts, R., Ide, S. y K. Ehlich (dirs.): Politeness in Language: Studies in its History, Theory and Practice. Berlin, Mouton de Gruyter.

Werkhofer, K. (1992): "Traditional and Modern Views: The Social Constitution of Politeness". En Watts R., Ide, S. y K. Ehlich (dirs.): Politeness in Language: Studies in its History and Practice. Berlín, Mouton de Gruyter, págs. 155-199.

Woodward, E. (1997): Lo formal y familiar en español e inglés. La Coruña, Universidade da Coruña. 